\title{
A modulation based phase difference estimator for real sinusoids to compensate for incoherent sampling
}

Kui Wang, Yaqing Tu, Yanlin Shen, Wei Xiao, and Des McLernon

Citation: Review of Scientific Instruments 89, 085120 (2018); doi: 10.1063/1.5026439

View online: https://doi.org/10.1063/1.5026439

View Table of Contents: http://aip.scitation.org/toc/rsi/89/8

Published by the American Institute of Physics

\section{Articles you may be interested in}

Mismatch error correction for time interleaved analog-to-digital converter over a wide frequency range Review of Scientific Instruments 89, 084709 (2018); 10.1063/1.5030782

A high-linearity time-to-digital converter based on dynamically delay-adjustable looped carry chains on FPGAs

Review of Scientific Instruments 89, 084704 (2018); 10.1063/1.5038146

Novel frequency stability measurement method with whole sample time range

Review of Scientific Instruments 89, 085113 (2018); 10.1063/1.5037928

A pico-second resolution arbitrary timing generator based on time folding and time interpolating Review of Scientific Instruments 89, 074701 (2018); 10.1063/1.5037841

A 90-102 GHz CMOS based pulsed Fourier transform spectrometer: New approaches for in situ chemical detection and millimeter-wave cavity-based molecular spectroscopy

Review of Scientific Instruments 89, 073109 (2018); 10.1063/1.5025729

Printed-circuit-board linear Paul trap for manipulating single nano- and microparticles

Review of Scientific Instruments 89, 083101 (2018); 10.1063/1.5007924

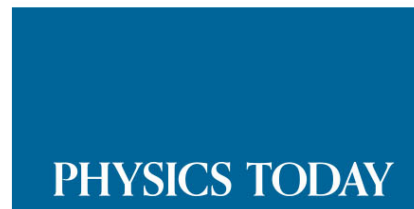

MANACER'S GUIDE

WHITEPAPERS
Accelerate R\&D with

Multiphysics Simulation

\section{READ NOW}

PRESENTED BY

$\checkmark \subset \bigcirc M S O L$ 


\title{
A modulation based phase difference estimator for real sinusoids to compensate for incoherent sampling
}

\author{
Kui Wang, ${ }^{1, a)}$ Yaqing Tu, ${ }^{1, a)}$ Yanlin Shen, ${ }^{2}$ Wei Xiao, ${ }^{1}$ and Des McLernon ${ }^{3}$ \\ ${ }^{1}$ Army Logistics University of PLA, Chongqing 401311, China \\ ${ }^{2}$ Logistics University of PAP, Tianjin 300309, China \\ ${ }^{3}$ The University of Leeds, Leeds LS2 9JT, United Kingdom
}

(Received 20 February 2018; accepted 1 August 2018; published online 27 August 2018)

\begin{abstract}
Phase difference estimation is a fundamental problem in numerous applications. However, incoherent sampling (IS) is an inevitable factor which degrades the precision of many correlations or Fourier transform-based approaches. In this paper, IS and the spectral superposition of real signals are both considered. A novel estimator is developed based on modulation and the discrete Fourier transform (DFT). With the estimated frequency, the phase difference can be obtained by calculating four DFT samples of the modulated signals. Simulations and the experimental results have proved their validity as well as their superiority over five other methods designed for IS, particularly at high signal-to-noise ratios. Furthermore, the proposed method can maintain high accuracy even when a significant bias occurs with the frequency estimation. Published by AIP Publishing. https://doi.org/10.1063/1.5026439
\end{abstract}

\section{INTRODUCTION}

The phase difference between two noisy versions of a sinusoid received at spatially separated sensors is an important parameter applied in tracking, positioning, direction finding, instrumentation, measurement, etc. One early similar application is estimating [via the discrete Fourier transform (DFT)] the direction of arrival (DOA) of signals incident on an array of antennas and overcoming the inherent estimation bias. ${ }^{1}$ For phase difference estimation, many approaches have been proposed so far. When the source signals are stationary, the generalized cross correlator $^{2}$ is a classical method which provides maximum likelihood estimation performance under the Gaussian signal and noise assumption. However, its resolution degrades at certain sampling rates. The approaches based on $\mathrm{DFT}^{3}$ and discrete-time-Fourier transform $(\mathrm{DTFT})^{4}$ calculate the phase difference directly through the subtraction of two DFT or DTFT phases at the signal frequency, but they suffer from spectrum leakage errors related to incoherent sampling (IS). For real sinusoids, the quadrature delay estimator (QDE), ${ }^{5}$ which utilizes in-phase and quadrature-phase components to get the estimated phase difference, is unbiased under the assumption of coherent sampling (CS). The correlation-based method $(\mathrm{CM})^{6,7}$ is computationally effective, but its performance is limited by the sampling period. On the other hand, for time-varying phase difference, online or adaptive techniques are widely concerned, ${ }^{8-11}$ such as the Hilbert transform (HT) method, ${ }^{10,11}$ which can estimate the rapidly changed phase difference. However, its precision is also influenced by the sampling period.

It is noticeable that in practical applications, IS always occurs because the varying signal frequency is usually sampled by using an analog-to-digital converter (ADC) at a fixed

a)Electronic addresses: yaqingtu@126.com and kuiwang.nudt@hotmail.com sampling rate. ${ }^{12}$ To some extent, a majority of estimators utilizing autocorrelation or cross-correlation exhibit the effect of bias according to IS, especially when the signal length is not sufficiently large. To solve this problem, the dataextension based correlation (DEC) method $^{13}$ directly extended the signal to approach CS by signal shift or truncation. The data-extension scheme was combined with the Hilbert transform (DEHT) in Ref. 14 to overcome the end effect of the HT method although data extension cannot exactly achieve CS even with a known frequency. Alternatively, the estimation bias caused by IS can be canceled out through analytical derivation. This can be found in the unbiased quadrature delay estimator (UQDE) ${ }^{15}$ and the phase and frequency matchingbased method. ${ }^{16}$ In the frequency domain, when the discrete sequence obtained by IS is processed within a DFT or DTFT window, the spectrum leakage error is appended to the estimated results. Such an error gets worse for real signals because of the spectral superposition of the positive- and negativefrequency components. For real sinusoids, the modified DTFT (M-DTFT)-based delay estimator ${ }^{15}$ converts the real signals to complex versions using the idea of the quadrature-phase component. Furthermore, the DTFT-based algorithms with negative frequency contribution (NFC-DTFT) ${ }^{17}$ significantly improve precision when the signal frequency is low or close to the Nyquist frequency. Further revisions of the NFC-DTFT method were constructed in Refs. 18-20 with window techniques and sliding algorithms to fit the time-varying signals. However, all the aforementioned methods designed for IS need a priori knowledge of actual signal's frequency, and they show bias when the accurate frequency information is unavailable.

The method proposed in this paper modulates the realvalued IS signals to approach CS, with the estimated frequency. The spectral superposition of the positive- and negativefrequency components is eliminated by some simple operations on the DFT samples. After all, the phase difference can 
be obtained by calculating only four DFT samples. In Sec. II, the general ideas, detailed derivation, and parameter settings of the estimator are presented. The simulations and experimental results are shown in Sec. III. The final conclusions are presented in Sec. IV.

\section{METHOD DEVELOPMENT}

\section{A. The underlying principle to tackle IS}

Consider the received signals of two noisy real sinusoids at the same frequency as follows:

$$
\left\{\begin{array}{l}
x_{1}(n)=s_{1}(n)+w_{1}(n)=A_{1} \cos \left(\omega_{0} n+\theta_{1}\right)+w_{1}(n) \\
x_{2}(n)=s_{2}(n)+w_{2}(n)=A_{2} \cos \left(\omega_{0} n+\theta_{2}\right)+w_{2}(n)
\end{array}, \quad n=0,1,2, \ldots, N-1,\right.
$$

where $A_{1}$ and $A_{2}$ are the amplitudes and $\theta_{1}$ and $\theta_{2}$ are the initial phases of the source signals $s_{1}(n)$ and $s_{2}(n) . \omega_{0}=2 \pi f / f_{s}$ $\left(0 \leq \omega_{0} \leq \pi\right)$ is the normalized frequency in radians, where $f$ is the actual signal frequency in $\mathrm{Hz}$ and $f_{s}$ is the sampling frequency in Hz. $w_{1}(n)$ and $w_{2}(n)$ are additive white Gaussian noises (AWGNs) with zero means and identical variance $\left(\sigma^{2}\right)$.

According to Euler's formula, the source signal $s_{i}(n), i=$ 1,2 can be rewritten as

$$
\begin{aligned}
s_{i}(n) & =A_{i} \cos \left(\omega_{0} n+\theta_{i}\right) \\
& =\left(A_{i} / 2\right) \cdot e^{j\left(\omega_{0} n+\theta_{i}\right)}+\left(A_{i} / 2\right) \cdot e^{-j\left(\omega_{0} n+\theta_{i}\right)} .
\end{aligned}
$$

If a priori knowledge of the signal frequency is assumed, the source signals can be adjusted to approach CS. Data extension provides a tangible solution, but the extended or truncated signal sequences cannot satisfy CS at various signal frequencies, and the errors get worse for short signals. Alternatively, modulation is more effective despite the signal length and frequency. Consider the modulated signal $x_{i, m}(n)$ as

$$
\begin{aligned}
x_{i, m}(n)= & \left(s_{i}(n)+w_{i}(n)\right) \cdot e^{-j n \omega_{m}} \\
= & s_{i, m}(n)+w_{i, m}(n) \\
= & \left(A_{i} / 2\right) \cdot e^{j\left(\left(\omega_{0}-\omega_{m}\right) n+\theta_{i}\right)} \\
& +\left(A_{i} / 2\right) \cdot e^{-j\left(\left(\omega_{0}+\omega_{m}\right) n+\theta_{i}\right)}+w_{i}(n) e^{-j n \omega_{m}},
\end{aligned}
$$

where $\omega_{m}$ is the modulation frequency in $\mathrm{rad} / \mathrm{s}$, which is proportional to $\omega_{0} \cdot s_{i, m}(n)$ and $w_{i, m}(n)$ represent the modulated source signal and noise. The modulated signal $x_{i, m}(n)$ results from a shift of the spectrum of $x_{i}(n)$ by $\omega_{m}$.

The DFT of $x_{i, m}(n)$, denoted as $X_{i, m}(k)$, is

$$
\begin{aligned}
X_{i, m}(k)= & \sum_{n=0}^{N-1}\left(s_{i, m}(n)+w_{i, m}(n)\right) \cdot e^{-j \frac{2 \pi}{N} n k} \\
= & S_{i, m}(k)+W_{i, m}(k) \\
= & \sum_{n=0}^{N-1}\left[\frac{A_{i}}{2} e^{j\left(\left(\omega_{0}-\omega_{m}-\frac{2 \pi k}{N}\right) n+\theta_{i}\right)}\right] \\
& +\sum_{n=0}^{N-1}\left[\frac{A_{i}}{2} e^{-j\left(\left(\omega_{0}+\omega_{m}+\frac{2 \pi k}{N}\right) n+\theta_{i}\right)}\right]+W_{i, m}(k),
\end{aligned}
$$

where $S_{i, m}(k)=\operatorname{DFT}\left[s_{i, m}(n)\right], W_{i, m}(k)=\operatorname{DFT}\left[w_{i, m}(n)\right]$, and $k=0,1,2, \ldots, N-1$.

In order to analyze the spectral superposition of real signals, the positive- and negative-frequency components of $S_{i, m}(k)$ are denoted as $S_{i, m P}(k)$ and $S_{i, m N}(k)$, respectively, where $S_{i, m P}(k)$ can be expressed as

$$
\begin{aligned}
S_{i, m P}(k) & =\sum_{n=0}^{N-1}\left[\frac{A_{i}}{2} e^{j\left(\left(\omega_{0}-\omega_{m}-\frac{2 \pi k}{N}\right) n+\theta_{i}\right)}\right]=\frac{A_{i}}{2} e^{j \theta_{i}} \sum_{n=0}^{N-1} e^{j\left(\omega_{0}-\omega_{m}-\frac{2 \pi k}{N}\right) n}=\frac{A_{i}}{2} e^{j \theta_{i}} \frac{1-e^{j\left(\omega_{0}-\omega_{m}-\frac{2 \pi k}{N}\right) N}}{1-e^{j\left(\omega_{0}-\omega_{m}-\frac{2 \pi k}{N}\right)}} \\
& =\frac{A_{i}}{2} e^{j \theta_{i}} \frac{e^{j \frac{N}{2}\left(\omega_{0}-\omega_{m}-\frac{2 \pi k}{N}\right)}\left(e^{-j\left(\omega_{0}-\omega_{m}-\frac{2 \pi k}{N}\right) \frac{N}{2}}-e^{j\left(\omega_{0}-\omega_{m}-\frac{2 \pi k}{N}\right) \frac{N}{2}}\right)}{e^{j \frac{1}{2}\left(\omega_{0}-\omega_{m}-\frac{2 \pi k}{N}\right)}\left(e^{-j \frac{1}{2}\left(\omega_{0}-\omega_{m}-\frac{2 \pi k}{N}\right)}-e^{j \frac{1}{2}\left(\omega_{0}-\omega_{m}-\frac{2 \pi k}{N}\right)}\right)} \\
& =\frac{A_{i}}{2} e^{j \theta_{i}} e^{j \frac{N-1)}{2}\left(\omega_{0}-\omega_{m}-\frac{2 \pi k}{N}\right)} \frac{\sin \left(\frac{N}{2}\left(\omega_{0}-\omega_{m}-\frac{2 \pi k}{N}\right)\right)}{\sin \left(\frac{1}{2}\left(\omega_{0}-\omega_{m}-\frac{2 \pi k}{N}\right)\right)},
\end{aligned}
$$

and similarly we can get

$$
\begin{aligned}
S_{i, m N}(k) & =\sum_{n=0}^{N-1}\left[\frac{A_{i}}{2} e^{-j\left(\left(\omega_{0}+\omega_{m}+\frac{2 \pi k}{N}\right) n+\theta_{i}\right)}\right] \\
& =\frac{A_{i}}{2} e^{-j \theta_{i}} e^{-j \frac{(N-1)}{2}\left(\omega_{0}+\omega_{m}+\frac{2 \pi k}{N}\right)} \frac{\sin \left(\frac{N}{2}\left(\omega_{0}+\omega_{m}+\frac{2 \pi k}{N}\right)\right)}{\sin \left(\frac{1}{2}\left(\omega_{0}+\omega_{m}+\frac{2 \pi k}{N}\right)\right)} .
\end{aligned}
$$

With a knowledge of $\omega_{0}$, the modulation frequency $\omega_{m}$ can be set to satisfy the following requirements:

$$
\left\{\begin{array}{l}
\left(\omega_{0}-\omega_{m}\right) N=2 q \pi \\
q=\operatorname{round}\left(\frac{\omega_{0} N}{2 \pi}\right)
\end{array},\right.
$$

where $q$ is a positive integer and the function of round( $(\cdot)$ means to round the number up or down to the nearest integer. 
According to this assumption, $\sin \left((N / 2)\left(\omega_{0}-\omega_{m}-2 \pi k / N\right)\right)$ $=0$ so that only if $k=q, S_{i, m P}(q) \neq 0$; otherwise, $S_{i, m P}(k)=0$. Then, Eq. (4) can be simplified as

$$
X_{i, m}(k)= \begin{cases}S_{i, m N}(k)+W_{i, m}(k), & k \neq q \\ S_{i, m N}(k)+S_{i, m P}(k)+W_{i, m}(k), & k=q .\end{cases}
$$

Ignoring the noisy term of $W_{i, m}(k)$, Fig. 1 shows an example of the discrete spectrum of the source signal $s_{i}(n)$ and the modulated signal $s_{i, m}(n)$ for $\omega_{0}=0.146 \pi$ and $N=45$. As is shown in Fig. 1(a), without modulation, the spectrum of the source signal shows obvious spectral superposition, which is detrimental for parameter estimation. Unfortunately, a majority of Fourier transform based estimators which were developed for complex signals or ignored the negative frequency contribution give a biased performance for real signals. After modulation [see Fig. 1(b)], the positive-frequency component $S_{i, m P}(k)$ shows no spectrum leakage, and the interaction of positive- and negative-frequency components can be separated in this case. That is to say, modulation is helpful to realize unbiased estimation for real sinusoids.

As we know, $S_{i, m P}\left(e^{j \omega}\right)=\operatorname{DTFT}\left[\left(A_{i} / 2\right) \cdot e^{j\left(\left(\omega_{0}-\omega_{m}\right) n+\theta_{i}\right)}\right]$ $=A_{i} \pi e^{j \theta_{i}} \delta\left(\omega-\left(\omega_{0}-\omega_{m}\right)\right)$, where $\delta(\cdot)$ is the Dirac delta function. The DFT of $\left(A_{i} / 2\right) \cdot e^{j\left(\left(\omega_{0}-\omega_{m}\right) n+\theta_{i}\right)}$ is of course the discrete sequence sampled from its DTFT with a frequency damping interval of $2 \pi / N$. From Eq. (7), we can find $\left.S_{i, m P}\left(e^{j \omega}\right)\right|_{\omega=2 \pi q / N}$ as the only DFT sample which is not equal to zero, and $\theta_{i}$ can be obtained directly by calculating the phase of $S_{i, m P}(q)$. Thus, the phase difference between $s_{1}(n)$ and $s_{2}(n)$ can be computed as

$$
\Delta \theta=\theta_{2}-\theta_{1}=\arctan \left(\frac{\operatorname{imag}\left(S_{2, m P}(q) / S_{1, m P}(q)\right)}{\operatorname{real}\left(S_{2, m P}(q) / S_{1, m P}(q)\right)}\right) .
$$

\section{B. Derivation of the estimator}

From the analysis above, the problem of calculating the phase difference converts to the estimation of $S_{i, m P}(q)$. From Eq. (8), $S_{i, m P}(q)$ can be obtained as

(a) Discrete spectrum of the source signal $s_{i}(n)$

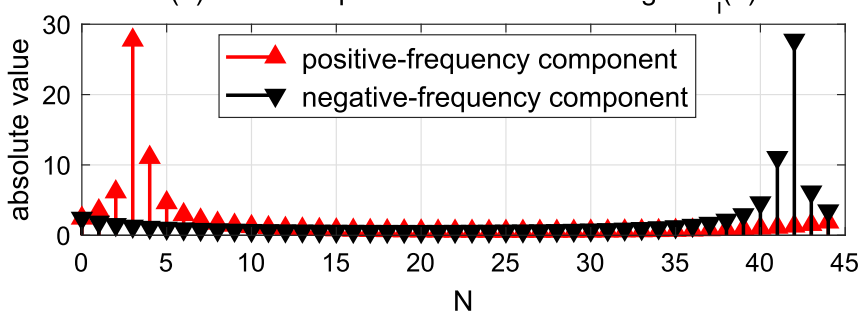

(b) Discrete spectrum of the modulated signal $s_{i, m}(n)$

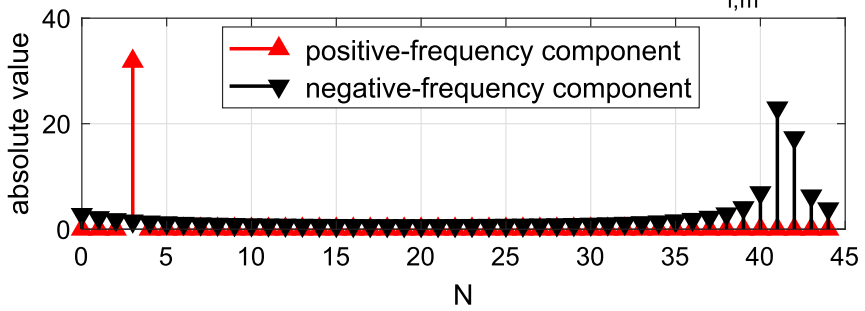

FIG. 1. Positive- and negative-frequency components of $s_{i}(n)$ and $s_{i, m}(n)$. (a) Discrete spectrum of the source signal $s_{i}(n)$. (b) Discrete spectrum of the modulated signal $s_{i, m}(n)$.

$$
S_{i, m P}(q)=X_{i, m}(q)-S_{i, m N}(q)-W_{i, m}(q) .
$$

Denoting $\Omega_{k}=\omega_{0}+\omega_{m}+2 \pi k / N$ for simplification and then from Eqs. (6) and (8), we obtain

$$
S_{i, m N}(q)=\frac{A_{i}}{2} e^{-j \theta_{i}} e^{-j \frac{(N-1)}{2} \Omega_{q}} \frac{\sin \left(N \Omega_{q} / 2\right)}{\sin \left(\Omega_{q} / 2\right)},
$$

$$
\begin{aligned}
X_{i, m}(k) & =S_{i, m N}(k)+W_{i, m}(k) \\
& =\frac{A_{i}}{2} e^{-j \theta_{i}} e^{-j \frac{(N-1)}{2} \Omega_{k}} \frac{\sin \left(N \Omega_{k} / 2\right)}{\sin \left(\Omega_{k} / 2\right)}+W_{i, m}(k), \quad k \neq q .
\end{aligned}
$$

Substitute Eqs. (11) and (12) into (10), and then $S_{i, m P}(q)$ can be obtained as

$$
\begin{aligned}
S_{i, m P}(q)= & X_{i, m}(q)-X_{i, m}(k) \frac{\sin \left(N \Omega_{q} / 2\right) \sin \left(\Omega_{k} / 2\right)}{\sin \left(\Omega_{q} / 2\right) \sin \left(N \Omega_{k} / 2\right)} \\
& \times e^{j \frac{(N-1)}{2}\left(\Omega_{k}-\Omega_{q}\right)}+\varepsilon(k), \quad k \neq q,
\end{aligned}
$$

where $\varepsilon(k)$ is the interference term caused by the AWGN,

$$
\begin{aligned}
\varepsilon(k)= & \frac{\sin \left(N \Omega_{q} / 2\right) \sin \left(\Omega_{k} / 2\right)}{\sin \left(\Omega_{q} / 2\right) \sin \left(N \Omega_{k} / 2\right)} e^{j \frac{(N-1)}{2}\left(\Omega_{k}-\Omega_{q}\right)} \\
& \times W_{i, m}(k)-W_{i, m}(q), \quad k \neq q .
\end{aligned}
$$

Because of $W_{i, m}(k)=\operatorname{DFT}\left[w_{i}(n) e^{-j n \omega_{m}}\right]$, it is easy to prove that $\mathrm{E}\left[W_{i, m}(k)\right]=0, \operatorname{var}\left[W_{i, m}(k)\right]=N \sigma^{2}$, and $\mathrm{E}\left[W_{i, m}(k) \cdot W_{i, m}^{*}(l)\right]=0$, when $k \neq l$. Accordingly, the expectation and variance of $\varepsilon(k)$ can be expressed as

$$
\begin{aligned}
\mathrm{E}[\varepsilon(k)]= & \frac{\sin \left(N \Omega_{q} / 2\right) \sin \left(\Omega_{k} / 2\right)}{\sin \left(\Omega_{q} / 2\right) \sin \left(N \Omega_{k} / 2\right)} e^{j \frac{(N-1)}{2}\left(\Omega_{k}-\Omega_{q}\right)} \\
& \times \mathrm{E}\left[W_{i, m}(k)\right]-\mathrm{E}\left[W_{i, m}(q)\right]=0, \quad k \neq q,
\end{aligned}
$$

$$
\begin{aligned}
\operatorname{var}[\varepsilon(k)] & =\mathrm{E}\left[|\varepsilon(k)|^{2}\right] \\
& =\left(\left(\frac{\sin \left(N \Omega_{q} / 2\right) \sin \left(\Omega_{k} / 2\right)}{\sin \left(\Omega_{q} / 2\right) \sin \left(N \Omega_{k} / 2\right)}\right)^{2}+1\right) \cdot N \sigma^{2} .
\end{aligned}
$$

Accordingly, an unbiased estimator of $S_{i, m P}(q)$ can be written as

$$
\begin{aligned}
\hat{S}_{i, m P}(q)= & X_{i, m}(q)-X_{i, m}(k) \frac{\sin \left(N \Omega_{q} / 2\right) \sin \left(\Omega_{k} / 2\right)}{\sin \left(\Omega_{q} / 2\right) \sin \left(N \Omega_{k} / 2\right)} \\
& \times e^{j \frac{(N-1)}{2}\left(\Omega_{k}-\Omega_{q}\right)}, \quad k \neq q .
\end{aligned}
$$

From Eq. (17), the estimator $\hat{S}_{i, m P}(q)$ needs to compute only two DFT samples as $X_{i, m}(q)$ and $X_{i, m}(k) . \Omega_{q}$ and $\Omega_{k}$ can then be obtained directly by the estimated signal frequency and the corresponding modulation frequency.

\section{Principle to choose $k$}

It should be noticed that in Eq. (17), $k$ can be an arbitrary integer varying from 0 to $N-1$ except $q$. Due to the nonlinear form of the phase of $\hat{S}_{i, m P}(q)$, it is difficult to find the analytical optimal integer $k$. However, we expect $\left|X_{i, m}(k)\right|$ to be large enough so that it would not be too sensitive to noise, where $|\cdot|$ means the absolute value. According to the rounding operation in Eq. (7), the peak value of $\left|X_{i, m}(k)\right|$ (excluding $\left.\left|X_{i, m}(q)\right|\right)$ usually occurs at $N-q-1, N-q$, or $N-q+1$. The frequency estimation error is another factor to be considered. 


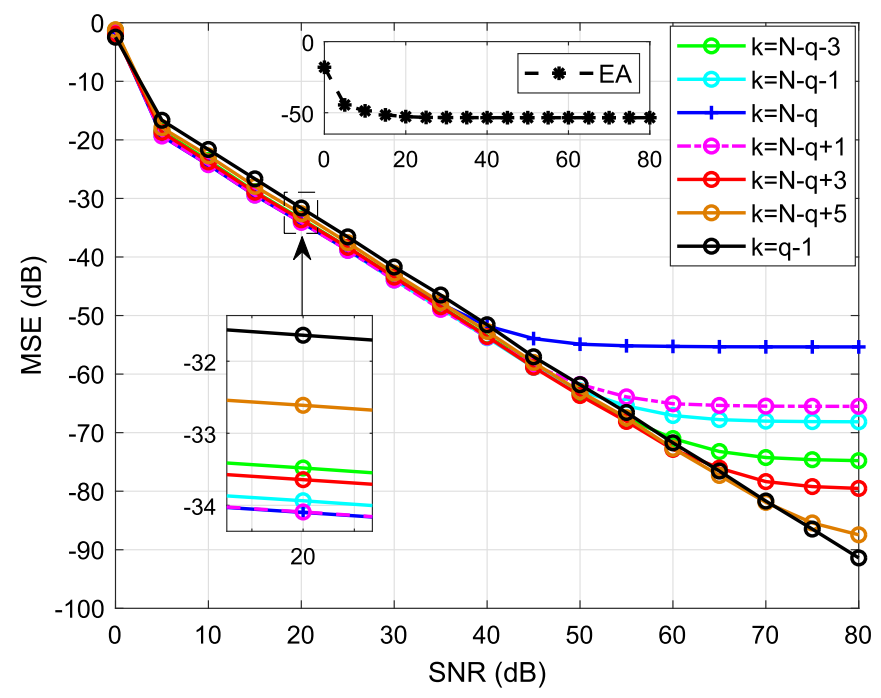

FIG. 2. Mean square phase difference errors versus SNR for different values of $k$ in Eq. (17) $\left(\omega_{0}=0.146 \pi, N=53\right)$.

The expanded autocorrelation (EA) method $^{21}$ is involved for frequency estimation, and it shows an obvious bias at high signal-to-noise ratios (SNRs). A typical example is given in Fig. 2. The performance of the EA method for frequency estimation is presented in the upper subgraph in Fig. 2, which shows mean-square errors (MSEs) saturation when the SNR is higher than $20 \mathrm{~dB}$. Seven different values of the integer $k$ [in Eq. (17)] are simulated at $\omega_{0}=0.146 \pi$ and $N=53$. Comparing their MSEs between two SNRs at $20 \mathrm{~dB}$ and $75 \mathrm{~dB}$, a "trade-off" effect can be found which generally indicates that the accuracy at high SNRs is always achieved at the cost of slightly reducing performance at low SNRs. In particular, if we set $k=q-1$, good precision can be guaranteed for high SNRs, but the method exhibits a bias at any value of SNR.

After a large number of simulations and numerical evaluations, an empirical principle of choosing $k$ was derived to set $k$ around $N-q$ at low SNRs when the accurate frequency information is available. Alternatively, if a significant bias happens to the frequency estimation, $k=q-1$ is not a bad choice to achieve high precision for high-SNR applications.

So the proposed method is summarized as follows:

(1) Use an estimated signal frequency $\hat{\omega}_{0}$ to replace $\omega_{0}$ in Eq. (7), and calculate the modulation frequency $\omega_{m}$ and the integer $q$.

(2) Modulate the source signal $s_{i}(n)$ to $s_{i, m}(n), i=1,2$ as in Eq. (3).

(3) Choose appropriate integer $k$ and calculate four DFT samples of the modulated signal including $X_{1, m}(q)$, $X_{1, m}(k), X_{2, m}(q)$, and $X_{2, m}(k)$.

(4) Obtain $\hat{S}_{1, m P}(q)$ and $\hat{S}_{2, m P}(q)$ by Eq. (17).

(5) Use Eq. (9) to realize the phase difference estimation.

\section{SIMULATIONS AND EXPERIMENTS}

\section{A. Simulations}

To verify the validity, comparative analysis based on simulations is conducted involving CM, HT, QDE, DTFT, DEC, DEHT, UQDE, M-DTFT, and NFC-DTFT approaches set

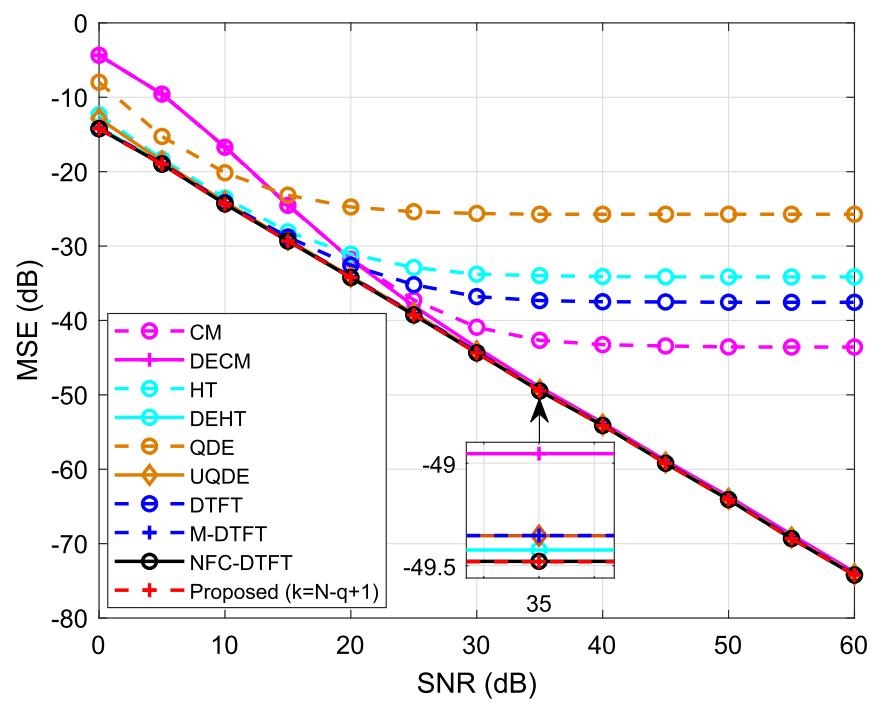

FIG. 3. Mean square phase difference errors versus $\operatorname{SNR}\left(\omega_{0}=\pi / 6, N=53\right)$.

against the proposed method. Without loss of generality, simulation parameters are set as $A_{1}=A_{2}=1, \Delta \theta=\pi / 6$, and $\theta_{2}=\theta_{1}+\Delta \theta$, while $\theta_{1}$ is uniformly distributed between $-\pi$ and $5 \pi / 6$. Each simulation result (including AWGN) is carried out with an average of 2000 independent runs.

Assuming the signal frequency $\omega_{0}=\pi / 6$ is known to the estimators, Fig. 3 shows the mean square phase difference errors versus SNR at $N=53$, which is recognized as an IS situation. Obviously, the conventional methods like CM, HT, QDE, and DTFT are biased estimators under IS. By contrast, the methods designed for IS including DEC, DEHT, UQDE, M-DTFT, and NFC-DTFT and the proposed method coincidently show high accuracy when the SNR is larger than $30 \mathrm{~dB}$, which has proved their effectiveness to overcome IS. From the subgraph, we can see that the performance of the proposed method and NFC-DTFT is even slightly better than those of the DEHT, UQDE, and M-DTFT methods.

When the signal frequency is unknown, the EA method is used to estimate the signal frequency, and the methods for IS

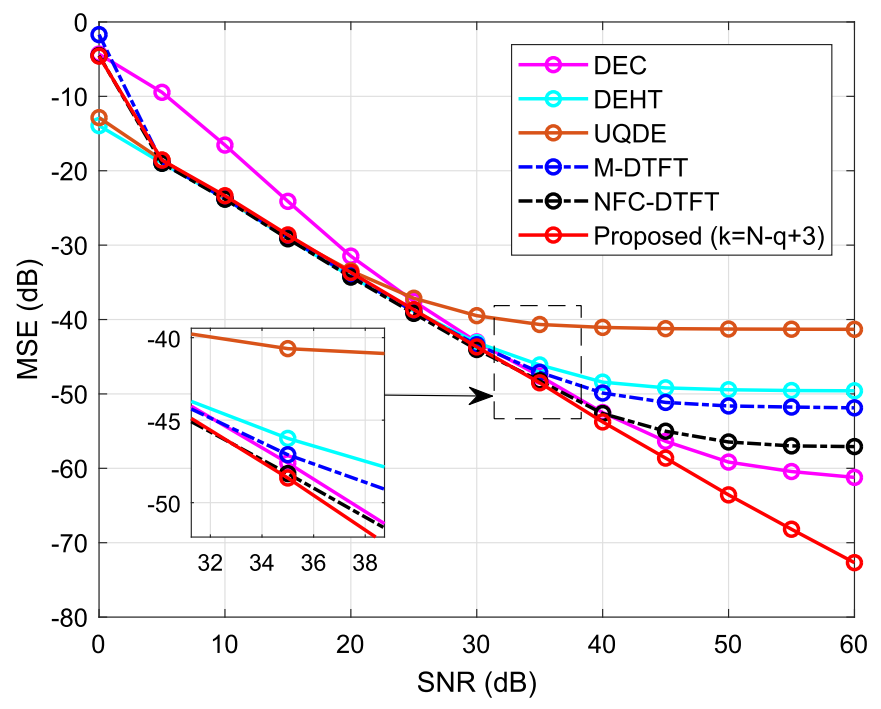

FIG. 4. Mean square phase difference errors versus SNR $\left(\omega_{0}=0.146 \pi\right.$, $N=53)$. 


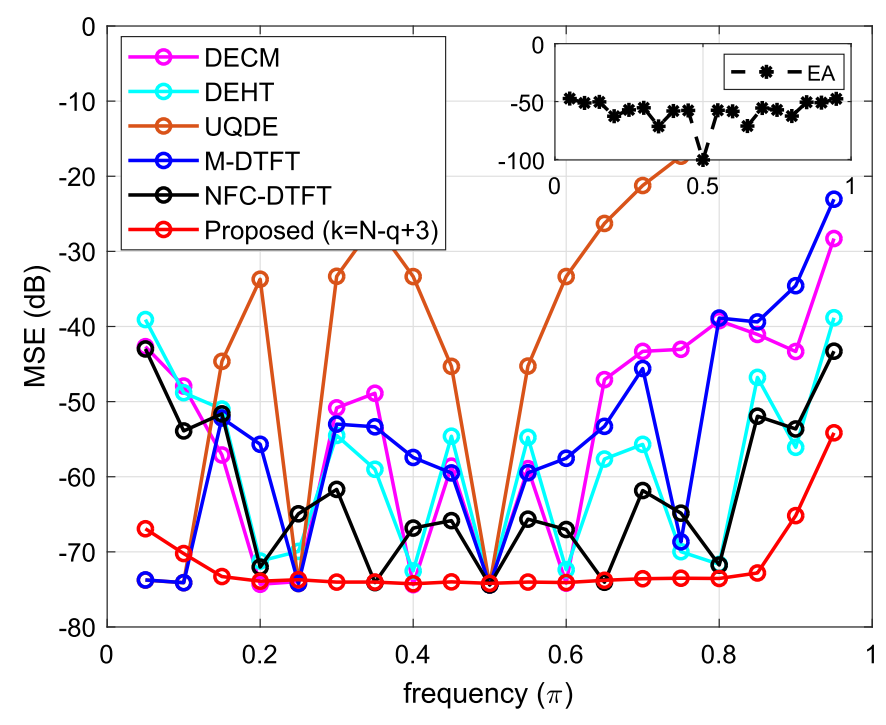

FIG. 5. Mean square phase difference errors versus frequency $(\mathrm{SNR}=60 \mathrm{~dB}$, $N=53)$.

suffer from the frequency estimation errors. The mean square phase difference errors versus SNR at $\omega_{0}=0.146 \pi$ and $N=53$ is shown in Fig. 4. In this case, $\pi /\left(2 \omega_{0}\right)$ is not equal to an integer so that the quadrature-phase component cannot be accurately obtained by time-shifting the signal by integer samples, and consequently, the UQDE and M-DTFT methods exhibit a bias when the SNR is higher than $30 \mathrm{~dB}$. Similarly, the extended signal sequences cannot exactly achieve CS at this signal frequency, and the performance of data-extension based methods such as DEC and DEHT degrades with the increase of SNR. As for NFC-DTFT and the proposed methods, their MSEs are mainly affected by the frequency estimation errors. However, the proposed method shows stronger robustness than the NFC-DTFT method, and it performs better than the other five approaches when the SNR is larger than $35 \mathrm{~dB}$.

To evaluate the precision at various signal frequencies, the mean square phase difference errors versus frequency at $\mathrm{SNR}=60 \mathrm{~dB}$ and $N=53$ is shown in Fig. 5. The signal frequency is estimated by the EA method, and its performance is provided as the dark dashed line in the upper right subgraph. In Fig. 5, the MSEs of the DEC, DEHT, M-DTFT, and UQDE methods fluctuate drastically with respect to variation of the signal frequency. They are affected by both the value and estimation errors of the signal frequency, and they reach the minimum only at certain points for meeting the CS requirement. In general, the NFC-DTFT and proposed methods exhibit better performance. The performance of the NFC-DTFT method is mainly affected by the frequency estimation errors, and the fluctuation of the dark line in Fig. 5 has the same characteristics as the line in the subgraph. For the proposed method, such an effect can also be found in calculating the angle of $\hat{S}_{i, m P}(q)$. However, due to the division in Eq. (9), the fluctuation effect is canceled out so that the proposed method can provide a steady and accurate estimation. Generally speaking, the proposed method shows higher anti-interference performance to variation of the signal frequency and relative estimation errors than the other five methods, and it has a high precision over a large range of the signal frequency.

\section{B. Measurement experiments}

To prove the validity in practice, a RHEONIK Coriolis mass flowmeter (CMF) with a RHE08 transmitter is used to test the performance of DEC, DEHT, UQDE, M-DTFT, and NFC-DTFT methods against the proposed method. In the experiments, the signal frequency $f$ is approximately $146 \mathrm{~Hz}$, and the sampling frequency $f_{s}$ is $2 \mathrm{kHz}$ so that the normalized frequency can be evaluated as $\omega_{0}=2 \pi f / f_{s}=0.146 \pi \approx$ $0.4587 \mathrm{rad}$. The flow rate varies from 0.40 to $16.72 \mathrm{~kg} \mathrm{~min}^{-1}$. In addition, 2650 points of sampled signals are divided into 50 segments, and for each segment, the signal length is $N=53$, which is the same as the parameter settings in simulations. The estimated time delay comes from an average of 50 segments. According to the measurement theory of CMF given in Ref. 22 , the mass flow rate $M_{f}$ is calculated as

$$
\left\{\begin{array}{l}
M_{f}=B_{0} \cdot \Delta t+C_{0} \\
\Delta t=\Delta \hat{\theta} /\left(\hat{\omega}_{0} \cdot f_{s}\right)
\end{array}\right.
$$

where $B_{0}=0.1392, C_{0}=-0.0052, \Delta \hat{\theta}$ is the estimated phase difference, $\Delta t$ is the corresponding time delay, and $\hat{\omega}_{0}$ is the estimated signal frequency by the EA method.

The experimental results are shown in Table I. The estimated frequency $\hat{\omega}_{0}$ fluctuates around the theoretical value $\omega_{0}=0.4587 \mathrm{rad}$, which contributes to the bias in the

TABLE I. Theoretical and estimated time delays under different flow rates.

\begin{tabular}{|c|c|c|c|c|c|c|c|c|c|}
\hline & \multirow[b]{2}{*}{ 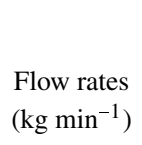 } & \multirow[b]{2}{*}{$\begin{array}{c}\text { Theoretical } \\
\text { Time delays }(\mu \mathrm{s})\end{array}$} & \multirow[b]{2}{*}{$\hat{\omega}_{0}(\mathrm{rad})$} & \multicolumn{6}{|c|}{ Estimated time delays $(\mu s)$} \\
\hline & & & & DEC & DEHT & UQDE & M-DTFT & NFC-DTFT & $\begin{array}{c}\text { Proposed } \\
(\mathrm{k}=\mathrm{N}-\mathrm{q}+3)\end{array}$ \\
\hline 1 & 0.40 & 2.902 & 0.4602 & 7.688 & 2.907 & 2.851 & 2.922 & 2.919 & 2.912 \\
\hline 2 & 1.12 & 8.107 & 0.4620 & 10.241 & 8.146 & 7.912 & 8.083 & 8.134 & 8.117 \\
\hline 3 & 4.32 & 31.061 & 0.4595 & 31.564 & 31.184 & 30.460 & 31.049 & 31.135 & 31.050 \\
\hline 6 & 10.14 & 72.859 & 0.4562 & 73.480 & 73.187 & 71.466 & 72.846 & 73.022 & 72.851 \\
\hline 7 & 12.10 & 86.950 & 0.4568 & 87.598 & 87.296 & 85.338 & 86.992 & 87.149 & 86.927 \\
\hline 8 & 13.95 & 100.282 & 0.4565 & 101.005 & 100.687 & 98.381 & 100.293 & 100.511 & 100.283 \\
\hline 9 & 16.72 & 120.126 & 0.4559 & 120.789 & 120.609 & 117.878 & 120.140 & 120.391 & 120.059 \\
\hline
\end{tabular}


later phase difference estimation. The average error rate for each phase difference estimator is calculated as $r_{i}=(1 / 9)$ $\times \sum_{i=1}^{9}\left(\Delta t_{i, \text { estimated }}-\Delta t_{i, \text { theoretical }}\right) / \Delta t_{i, \text { theoretical }}, i=1,2, \ldots, 9$, and the results of DEC, DEHT, UQDE, M-DTFT, and NFCDTFT methods and the proposed method are $22.00 \%, 0.40 \%$, $1.93 \%, 0.14 \%, 0.28 \%$, and $0.07 \%$, respectively. Thus, the superiority of the proposed method is demonstrated by these experimental measurements.

\section{CONCLUSION}

To tackle the bias caused by IS, an accurate phase difference estimator is proposed based on modulation and the DFT. The estimated results can be obtained by calculating four DFT samples of the modulated signals. Unlike data-extension based methods, our proposed method can approach CS at various signal lengths and frequencies. The spectral superposition of real sinusoids is eliminated which is helpful to achieve unbiased estimation. Compared with DEC, DEHT, UQDE, M-DTFT, and NFC-DTFT methods, the proposed method shows higher anti-interference performance to frequency estimation errors, which enables it to provide reliable results when significant errors happen to the frequency estimation. The simulation results have proved its superiority, particularly at high SNRs. The validity of the proposed method is also demonstrated by field experiments. Finally, the proposed method is also capable of estimating the signal amplitude and initial phase.

\section{ACKNOWLEDGMENTS}

The study is supported by the National Natural Science Foundation of China (NNSFC) (Grant Nos. 61271449 and 61302175), postgraduate research innovation project of Chongqing (CYB14100), Natural Science Foundation of Tianjin (Grant No. 18JCQNJC01400), Key Program of Natural Science Foundation of Chongqing (Grant No. cstc2015 jcyjBX0017), Ph.D. startup foundation (WHB201707), and Special Project for 100 Academic and Discipline Talents of Chongqing (Grant No. 2012-44).

${ }^{1}$ M. Zatman, D. C. McLernon, M. MacLeod, and H. Strangeways, "Estimation bias variation on beamformed signals," IEEE Trans. Antennas Propag. 43, 1496-1499 (1995).

${ }^{2}$ C. H. Knapp and G. C. Carter, "The generalized correlation method foe estimation of time delay," IEEE Trans. Acoust., Speech, Signal Process. 24, 320-327 (1976).
${ }^{3}$ P. Romano, "Coriolis mass flow rate meter having a substantially increased noise immunity," U.S. patent specification 4934196 (June 19, 1990).

${ }^{4}$ H. C. So, "Two discrete-time phase delay estimators," in International Symposium on Signal Processing and its Applications (IEEE, 2003), Vol. 2, pp. 547-550.

${ }^{5}$ D. L. Maskell and G. S. Woods, "The discrete-time quadrature subsample estimation of delay," IEEE Trans. Instrum. Meas. 51, 133-137 (2002).

${ }^{6}$ A. K. Nandi, "On the subsample time delay estimation of narrowband ultrasonic echoes,” IEEE Trans. Ultrason., Ferroelectr. Freq. Control 42, 993-1001 (1995).

${ }^{7}$ J. Li, P. Zhao, W. Hou, B. Zheng, and H. Zheng, "Research on error of phase difference algorithm based on correlation theory," J. North Univ. China 30, 616-629 (2009).

${ }^{8}$ M. Chakraborty, H. C. So, and J. Zheng, "New adaptive algorithm for delay estimation of sinusoidal signals," IEEE Signal Process. Lett. 14, 984-987 (2007).

${ }^{9} \mathrm{M}$. Chakraborty, "A new adaptive filter for estimating and tracking the delay and the amplitude of a sinusoid," IEEE Trans. Instrum. Meas. 59, 3049-3057 (2010).

${ }^{10}$ H. Yang, Y. Tu, H. Zhang, and P. Yi, "Phase difference measuring method based on SVD and Hilbert transform for Coriolis mass flowmeter," Chin. J. Sci. Instrum. 33, 2101-2107 (2012).

${ }^{11}$ W. Liu, L. Zhao, K. Wang, Z. Feng, and Q. Long, "Signal processing for Coriolis mass flowmeter based on Hilbert transform," Acta Metrol. Sin. 31, 446-451 (2013).

${ }^{12}$ T. Yamada, "High-accuracy estimations of frequency, amplitude, and phase with a modified DFT for asynchronous sampling," IEEE Trans. Instrum. Meas. 62, 1428-1435 (2013).

${ }^{13}$ T. Shen, Y. Tu, M. Li, and H. Zhang, "Research and validation on improved correlation method for phase difference measurement based on data extension," Chin. J. Sci. Instrum. 35, 1331-1337 (2014).

${ }^{14}$ Y. Shen, Y. Tu, L. Chen, and T. Shen, "Phase difference estimation method based on data extension and Hilbert transform," Meas. Sci. Technol. 26, 095003 (2015).

${ }^{15}$ H.C. So, "A comparative study of two discrete-time phase delay estimators," IEEE Trans. Instrum. Meas. 54, 2501-2504 (2005).

${ }^{16} \mathrm{Y}$. Tu, Y. Shen, H. Zhang, and M. Li, "Phase and frequency matching-based signal processing method for Coriolis mass flowmeters," Meas. Sci. Rev. 16, 62-67 (2016).

${ }^{17} \mathrm{Y}$. Tu and H. Zhang, "Method for CMF signal processing based on the recursive DTFT algorithm with negative frequency contribution," IEEE Trans. Instrum. Meas. 57, 2647-2654 (2008).

${ }^{18}$ T. Shen, Y. Tu, M. Li, and H. Zhang, "A new phase difference measurement algorithm for extreme frequency signals based on discrete time Fourier transform with negative frequency contribution," Rev. Sci. Instrum. 86, 015104 (2015).

${ }^{19}$ T. Shen, Y. Tu, and H. Zhang, "A novel time varying signal processing method for Coriolis mass flowmeter," Rev. Sci. Instrum. 85, 065116 (2014).

${ }^{20} \mathrm{Y}$. Tu, T. Shen, H. Zhang, and M. Li, "Two new sliding DTFT algorithms for phase difference measurement based on a new kind of windows," Meas. Sci. Rev. 14, 350-356 (2014).

${ }^{21}$ Y. Cao, G. Wei, and F. Chen, "A closed-form expanded autocorrelation method for frequency estimation of a sinusoid," Signal Process. 92, 885-892 (2012).

${ }^{22}$ Y. Shen and Y. Tu, "Correlation theory-based signal processing method for CMF signals," Meas. Sci. Technol. 27, 065006 (2016). 